\title{
GRAPH VARIETIES AXIOMATIZED BY SEMIMEDIAL, MEDIAL, AND SOME OTHER GROUPOID IDENTITIES
}

\author{
ERKKO LEHTONEN \\ Technische Universität Dresden \\ Institut für Algebra \\ 01062 Dresden, Germany \\ e-mail: Erkko.Lehtonen@tu-dresden.de \\ AND \\ Chaowat Manyuen \\ Department of Mathematics, Faculty of Science \\ Khon Kaen University \\ Khon Kaen 40002, Thailand \\ e-mail: schauwat.manyuen@gmail.com
}

\begin{abstract}
Directed graphs without multiple edges can be represented as algebras of type $(2,0)$, so-called graph algebras. A graph is said to satisfy an identity if the corresponding graph algebra does, and the set of all graphs satisfying a set of identities is called a graph variety. We describe the graph varieties axiomatized by certain groupoid identities (medial, semimedial, autodistributive, commutative, idempotent, unipotent, zeropotent, alternative).
\end{abstract}

Keywords: graph algebra, groupoid, identities, semimediality, mediality. 2010 Mathematics Subject Classification: 05C25, 03C05.

\section{REFERENCES}

[1] B.A. Davey, P.M. Idziak, W.A. Lampe and G.F. McNulty, Dualizability and graph algebras, Discrete Math. 214 (2000) 145-172. doi:10.1016/S0012-365X(99)00225-3

[2] K. Denecke, M. Erné and S.L. Wismath (eds.), Galois connections and applications, Math. Appl., vol. 565 (Kluwer Academic Publishers, Dordrecht, 2004). doi:10.1007/978-1-4020-1898-5 
[3] E.W. Kiss, R. Pöschel and P. Pröhle, Subvarieties of varieties generated by graph algebras, Acta Sci. Math. (Szeged) 54 (1990) 57-75.

[4] T. Poomsa-ard, Hyperidentities in associative graph algebras, Discuss. Math. Gen. Algebra Appl. 20 (2000) 169-182. doi:10.7151/dmgaa.1014

[5] T. Poomsa-ard and W. Hemvong, Hyperidentities in left self-distributive graph algebras, Thai J. Math. 4 (2006) 197-208.

[6] T. Poomsa-ard and W. Hemvong, Hyperidentities in right self-distributive graph algebras of type (2,0), Southeast Asian Bull. Math. 32 (2008) 1125-1136.

[7] R. Pöschel, The equational logic for graph algebras, Z. Math. Logik Grundlag. Math. 35 (1989) 273-282. doi:10.1002/malq.19890350311

[8] R. Pöschel, Graph algebras and graph varieties, Algebra Universalis 27 (1990) 559577. doi:10.1007/BF01189000

[9] R. Pöschel and W. Wessel, Classes of graphs definable by graph algebra identities or quasi-identities, Comment. Math. Univ. Carolin. 28 (1987) 581-592. http://dml. cz/handle/10338.dmlcz/106570

[10] C.R. Shallon, Non-finitely based binary algebras derived from lattices, Ph.D. thesis, (University of California, Los Angeles, 1979).

Submitted 10 October 2018

First Revised 6 December 2018

Second Revised 6 June 2019

Accepted 6 December 2019 